\title{
ON SUMMABILITY CONDITIONS FOR INTERVAL MAPS
}

\author{
HUAIBIN LI
}

(Received 23 March 2013; accepted 7 April 2013; first published online 13 June 2013)

\begin{abstract}
Consider a map of class $C^{3}$ with nonflat critical points and with all periodic points hyperbolic repelling. We show that the 'backward contracting condition' implies the summability condition. This result is the converse of Theorem 3 of Bruin et al. ['Large derivatives, backward contraction and invariant densities for interval maps', Invent. Math. 172 (2008), 509-533].
\end{abstract}

2010 Mathematics subject classification: primary 37E05.

Keywords and phrases: interval maps, summability condition, backward contracting.

\section{Introduction}

Let $I$ be a compact interval of $\mathbb{R}$. For a differentiable map $f: I \rightarrow I$, a point of $I$ is critical if the derivative of $f$ vanishes at it. Denote by $\operatorname{Crit}(f)$ the set of critical points of $f$, and put $\mathrm{CV}(f):=f(\operatorname{Crit}(f))$. As usual, we say that a differentiable interval map $f: I \rightarrow I$ is of class $C^{3}$ with nonflat critical points, if it has a finite number of critical points and if:

- $\quad$ the map $f$ is of class $C^{3}$ outside $\operatorname{Crit}(f)$;

- $\quad$ for each critical point $c$ of $f$ there exist a number $\ell_{c}>1$ and diffeomorphisms $\phi$ and $\psi$ of $\mathbb{R}$ of class $C^{3}$, such that $\phi(c)=\psi(f(c))=0$, and such that in a neighbourhood of $c$ on $I$, we have $|\psi \circ f|=|\phi|^{\ell_{c}}$.

Let $f: I \rightarrow I$ be a map of class $C^{3}$ with nonflat critical points. For an integer $n \geq 1$, a periodic point $p$ of $f$ of period $n$ is hyperbolic repelling if $\left|D f^{n}(p)\right|>1$. Throughout the rest of this paper, we denote by $\mathscr{A}$ the collection of interval maps of class $C^{3}$ with nonflat critical points and with all periodic points hyperbolic repelling.

Definition 1.1. Given $\beta>0$, we say that a map $f \in \mathscr{A}$ satisfies the summability condition with exponent $\beta$, if for every $c \in \operatorname{Crit}(f)$ we have

$$
\sum_{n=0}^{+\infty}\left|D f^{n}(f(c))\right|^{-\beta}<+\infty .
$$

The author was supported by the National Natural Science Foundation of China (Grant No. 11101124).

(C) 2013 Australian Mathematical Publishing Association Inc. 0004-9727/2013 \$16.00 
This type of condition was first posed by Nowicki and van Strien in their work [5] on dynamics of unimodal interval maps. It plays an important role in the study of one-dimensional dynamics. For example, it is weaker than the Collet-Eckmann condition [2], but still guarantees the existence of an absolutely continuous invariant measure.

In [6], Rivera-Letelier first introduced a new notion called the backward contracting condition for rational maps, which serves as a different type of nonuniform hyperbolicity condition, and then it was adapted to interval maps in [1]. This condition is more convenient to use as it follows immediately that the first return maps to suitably chosen small neighbourhoods of critical points have good combinatorial and geometric properties. For instance, this notion plays an important role in [1, 3].

Given a map $f \in \mathscr{A}$, denote by $\operatorname{dom}(f)$ the interval on which $f$ is defined, and denote by dist the distance on $\operatorname{dom}(f)$ induced by the norm distance on $\mathbb{R}$. We shall use $\ell_{c}$ to denote the order of $c \in \operatorname{Crit}(f)$, and use $\ell_{\max }(f)$ to denote the maximal order of critical points.

For every $c \in \operatorname{Crit}(f)$ and $\delta>0$, let $\widetilde{B}(c, \delta):=B\left(c, \delta^{1 / \ell_{c}}\right)$ and put

$$
\widetilde{B}(\delta):=\bigcup_{c \in \operatorname{Crit}(f)} \widetilde{B}(c, \delta) .
$$

Definition 1.2. Given $\delta_{0}>0$ and a function $\mathbf{r}:\left(0, \delta_{0}\right) \rightarrow(1,+\infty)$, we say that $f$ is backward contracting with function $\mathbf{r}$, if for every $\delta \in\left(0, \delta_{0}\right)$, every $c \in \operatorname{Crit}(f)$, every integer $n \geq 0$ and every component $W$ of $f^{-n}(\widetilde{B}(c, \mathbf{r}(\delta) \delta))$,

$$
\operatorname{dist}(W, \mathrm{CV}(f)) \leq \delta \text { implies }|W|<\delta .
$$

Moreover, if the function $\mathbf{r}$ also satisfies $\mathbf{r}(\delta) \geq r$ for some $r>1$ and all sufficiently small $\delta>0$, then we say that $f$ is backward contracting with constant $r(f \in \mathrm{BC}(r)$ in short). If $f \in \mathrm{BC}(r)$ for all $r>1$, we write $f \in \mathrm{BC}(\infty)$.

In [1], the authors proved the following result.

Proposition 1.3 [1, Theorem 3]. Take $f \in \mathscr{A}$ and $\beta>0$. If $f$ satisfies the summability condition with exponent $\beta$, then there exist $\delta_{0}>0$ and a function $\mathbf{r}:\left(0, \delta_{0}\right) \rightarrow(1,+\infty)$ such that, for every $\theta \in(0,1)$,

$$
\sum_{n \gg 1} \mathbf{r}\left(\theta^{n}\right)^{-\beta}<+\infty,
$$

and such that $f$ is backward contracting with function $\mathbf{r}$.

The main goal of this note is to prove the following theorem.

Theorem 1.4. Let $f$ be a map in $\mathscr{A}$, and let $\beta>0$. Assume that there exist $\delta_{0}>0$ and a function $\mathbf{r}:\left(0, \delta_{0}\right) \rightarrow(1,+\infty)$ such that, for some $\theta_{0} \in(0,1)$,

$$
\sum_{n \gg 1} \mathbf{r}\left(\theta_{0}^{n}\right)^{-\beta}<+\infty,
$$


and such that $f$ is backward contracting with function $\mathbf{r}$. Then $f \in \mathrm{BC}(\infty)$ and $f$ satisfies the summability condition with exponent $\beta$.

Combining Proposition 1.3 and Theorem 1.4, we obtain the following corollary.

Corollary 1.5. Let $f$ be a map in $\mathscr{A}$, and let $\beta>0$. Then $f$ satisfies the summability condition with exponent $\beta$ if and only if there exist $\delta_{0}>0$ and a function $\mathbf{r}:\left(0, \delta_{0}\right) \rightarrow$ $(1,+\infty)$ such that, for some $\theta_{0} \in(0,1)$,

$$
\sum_{n \gg 1} \mathbf{r}\left(\theta_{0}^{n}\right)^{-\beta}<+\infty,
$$

and such that $f$ is backward contracting with function $\mathbf{r}$.

Let us say that an interval map $f \in \mathscr{A}$ is of Misiurewicz type, if for each $c \in \operatorname{Crit}(f)$ we have $\omega(c) \cap \operatorname{Crit}(f)=\emptyset$, where $\omega(c)$ is the omega-limit set of the orbit of $c$. This includes the case where $\operatorname{Crit}(f)=\emptyset$. For maps of Misiurewicz type, our theorem is well known or trivial. So, throughout the rest of this paper, fix an interval map $f \in \mathscr{A}$, and assume that $f$ is not of Misiurewicz type.

\section{Preliminaries}

We shall use the following version of the Koebe distortion theorem which was proved in [1].

Lemma 2.1 [1, Proposition 1(3)]. For any $f \in \mathscr{A}$, there exists $\eta(f)>0$ such that the following result holds. Let $s \geq 1$ be an integer and let $T=(a, b)$ be a subinterval of $\operatorname{dom}(f)$. Assume that $\left|f^{s}(T)\right|<\eta(f)$ and that $f_{\mid T}^{s}$ is a diffeomorphism onto its image. If $J$ is a subinterval of $T$ such that both components of $f^{s}(T) \backslash f^{s}(J)$ have length at least $\tau\left|f^{s}(J)\right|$ for some $\tau>0$, then for any $x, y \in J$,

$$
0.9\left(\frac{\tau}{1+\tau}\right)^{2} \leq \frac{\left|D f^{s}(x)\right|}{\left|D f^{s}(y)\right|} \leq \frac{1}{0.9}\left(\frac{1+\tau}{\tau}\right)^{2}
$$

Given a set $V$, we say that $W$ is a pull-back of $V$, if there exists an integer $s \geq 0$ such that $W$ is a connected component of $f^{-s}(V)$. For every $\delta>0$, let $\mathscr{P}(\delta)$ be the collection of all pull-backs of $\widetilde{B}(\delta)$ that contain a critical value, and define

$$
\Gamma(\delta):=\inf \left\{\frac{\delta}{|V|}: V \in \mathscr{P}(\delta)\right\} .
$$

Note that our standard assumption that $f$ is not of Misiurewicz type implies, for every $\delta>0$, that $\Gamma(\delta)$ is well defined. For this function we have the following properties.

Lemma 2.2. For any $\delta_{1} \geq \delta_{2}>0$, we have $\delta_{1} / \Gamma\left(\delta_{1}\right) \geq \delta_{2} / \Gamma\left(\delta_{2}\right)$. 
Proof. Observe that for every $V \in \mathscr{P}\left(\delta_{2}\right)$ there is $\widehat{V} \in \mathscr{P}\left(\delta_{1}\right)$ such that $V \subset \widehat{V}$. It follows that

$$
\begin{aligned}
\Gamma\left(\delta_{2}\right) & =\inf \left\{\frac{\delta_{2}}{|V|}: V \in \mathscr{P}\left(\delta_{2}\right)\right\}=\frac{\delta_{2}}{\delta_{1}} \inf \left\{\frac{\delta_{1}}{|V|}: V \in \mathscr{P}\left(\delta_{2}\right)\right\} \\
& \geq \frac{\delta_{2}}{\delta_{1}} \inf \left\{\frac{\delta_{1}}{|\widehat{V}|}: \widehat{V} \in \mathscr{P}\left(\delta_{1}\right)\right\}=\frac{\delta_{2}}{\delta_{1}} \Gamma\left(\delta_{1}\right) .
\end{aligned}
$$

This implies that $\delta_{1} / \Gamma\left(\delta_{1}\right) \geq \delta_{2} / \Gamma\left(\delta_{2}\right)$. The lemma is proved.

Lemma 2.3 [4, Theorem A.1]. Take $\beta>0$ and $f \in \mathscr{A}$. If there is $\delta_{0}>0$ such that

$$
\int_{0}^{\delta_{0}} \Gamma(t)^{-\beta} \frac{d t}{t}<+\infty
$$

then $f$ satisfies the summability condition with exponent $\beta$.

Lemma 2.4. Take $f \in \mathscr{A}$ and $\beta>0$. If there exists $\theta \in(0,1)$ such that

$$
\sum_{n=1}^{+\infty} \Gamma\left(\theta^{n}\right)^{-\beta}<+\infty
$$

then $f$ satisfies the summability condition with exponent $\beta$.

Proof. By Lemma 2.2 for every integer $n \geq 1$ and every $\delta \in\left[\theta^{n+1}, \theta^{n}\right]$,

$$
\frac{\theta^{n+1}}{\Gamma\left(\theta^{n+1}\right)} \leq \frac{\delta}{\Gamma(\delta)} \leq \frac{\theta^{n}}{\Gamma\left(\theta^{n}\right)}
$$

which implies that

$$
\theta^{\beta} \Gamma\left(\theta^{n+1}\right)^{-\beta} \leq \Gamma(\delta)^{-\beta} \leq \theta^{-\beta} \Gamma\left(\theta^{n}\right)^{-\beta} .
$$

Thus

$$
\Gamma\left(\theta^{n+1}\right)^{-\beta} \theta^{\beta} \log \theta^{-1} \leq \int_{\theta^{n+1}}^{\theta^{n}} \Gamma(t)^{-\beta} \frac{d t}{t} \leq \Gamma\left(\theta^{n}\right)^{-\beta} \theta^{-\beta} \log \theta^{-1} .
$$

This implies that

$$
\int_{0}^{\theta} \Gamma(t)^{-\beta} \frac{d t}{t}<+\infty .
$$

The assertion of the lemma follows from Lemma 2.3.

For every $\delta>0$, define

$$
\gamma(\delta):=\sup \{r>1: \text { if } V \in \mathscr{P}(r \delta), \text { then }|V|<\delta\} .
$$

Observe that if $f$ is backward contracting with some function $\mathbf{r}$, then there is $\delta_{0}>0$ such that the function $\gamma$ is well defined on $\left(0, \delta_{0}\right]$. Throughout the rest of this section, we will let $\delta^{*}>0$ be such that $\gamma(\delta)$ is well defined on $\left(0, \delta^{*}\right]$. 
Lemma 2.5. For any $\delta_{1} \geq \delta_{2}$ in $\left(0, \delta^{*}\right]$, we have $\gamma\left(\delta_{1}\right) \delta_{1} \geq \gamma\left(\delta_{2}\right) \delta_{2}$.

Proof. By the definition of $\gamma(\delta)$, every pull-back $V$ of $\widetilde{B}\left(\gamma\left(\delta_{2}\right) \delta_{2}\right)$ that contains a critical value satisfies $|V| \leq \delta_{2}$. In other words, every pull-back $V$ of $\widetilde{B}\left(\left(\gamma\left(\delta_{2}\right) \delta_{2} / \delta_{1}\right) \delta_{1}\right)$ that contains a critical value satisfies $|V| \leq \delta_{2} \leq \delta_{1}$. Using the definition of $\gamma(\delta)$ again, we know that $\gamma\left(\delta_{1}\right) \geq \gamma\left(\delta_{2}\right) \delta_{2} / \delta_{1}$. This implies that $\gamma\left(\delta_{1}\right) \delta_{1} \geq \gamma\left(\delta_{2}\right) \delta_{2}$. The lemma is proved.

Lemma 2.6 [1, Lemma 2]. Take $r>1$ and let $f \in \mathscr{A}$. If there is $\delta_{0} \in\left(0, \delta^{*}\right]$ such that for each $\delta \in\left(0, \delta_{0}\right]$ we have $\gamma(\delta) \geq 8^{\ell_{\max }} r$, then $f \in \mathrm{BC}(r)$.

Lemma 2.7. Let $\beta>0$ and $\theta \in\left(0, \delta^{*}\right)$. If $\sum_{n=1}^{+\infty} \gamma\left(\theta^{n}\right)^{-\beta}<+\infty$, then $f \in \mathrm{BC}(\infty)$.

Proof. In view of Lemma 2.6, it suffices to show that for every $r>1$ there is $\delta_{0}>0$ such that, for every $\delta \in\left(0, \delta_{0}\right]$, we have $\gamma(\delta) \geq 8^{\ell_{\max }} r$. Fix $r>1$. By assumption there exists $N$ such that, for every integer $n \geq N$, we have $\gamma\left(\theta^{n}\right) \geq 8^{\ell_{\max }} \theta^{-1} r$. Let $\delta_{0}=\theta^{N}$. For any $\delta \in\left(0, \delta_{0}\right]$ there is an integer $s(\delta) \geq N$ such that

$$
\theta^{s(\delta)+1}<\delta \leq \theta^{s(\delta)}
$$

It follows by Lemma 2.5 that

$$
\gamma(\delta) \geq \frac{\theta^{s(\delta)+1} \gamma\left(\theta^{s(\delta)+1}\right)}{\delta} \geq \frac{\theta^{s(\delta)+1} \gamma\left(\theta^{s(\delta)+1}\right)}{\theta^{s(\delta)}} \geq \theta 8^{\ell_{\max }} \theta^{-1} r=8^{\ell_{\max }} r .
$$

The proof is complete.

Lemma 2.8. Suppose that $f \in \mathrm{BC}(r)$ for some $r>1$. Then there exists $\tau_{0}>0$ such that, for every $\tau \in\left(0, \tau_{0}\right)$, the following result holds. For each integer $s \geq 1$ and any connected component $V$ of $f^{-s}(\widetilde{B}(\tau))$ containing a critical value, there exist an integer $1 \leq s^{\prime} \leq s, c \in C r i t(f)$ and a connected component $V^{\prime}$ of $f^{-s^{\prime}}(\widetilde{B}(c, \tau))$ containing $V$ such that $f^{s^{\prime}}: V^{\prime} \rightarrow \widetilde{B}(c, \tau)$ can be extended diffeomorphically onto $\widetilde{B}(c, r \tau)$.

Proof. By assumption $f \in \mathrm{BC}(r)$, there is $\tau_{0}>0$ such that, for each $\tau \in\left(0, \tau_{0}\right]$ and each $V \in \mathscr{P}(r \tau)$, we have $|V|<\tau$. Reducing $\tau_{0}$ if necessary, we assume in addition that $\tau_{0}<\operatorname{dist}(\operatorname{Crit}(f), C V(f))$. Fix $\tau \in\left(0, \tau_{0}\right]$, and let $V \in \mathscr{P}(\tau)$. Therefore there exist $c_{*} \in \operatorname{Crit}(f)$ and an integer $s \geq 1$ such that $V$ is the connected component of $f^{-s}\left(\widetilde{B}\left(c_{*}, \tau\right)\right)$. If $s=1$, by our choice of $\tau_{0}$ the assertion of the lemma is obvious. Hence, in the following we will assume that $s \geq 2$.

Let $\widetilde{V}$ be the connected component of $f^{-s}\left(\widetilde{B}\left(c_{*}, r \tau\right)\right)$ containing $V$. If $f^{s}: \widetilde{V} \rightarrow$ $\widetilde{B}\left(c_{*}, r \tau\right)$ is diffeomorphic, then we complete the proof with $s^{\prime}=s, c=c_{*}$ and $V^{\prime}=V$. Otherwise, there exists an integer $s^{*} \in[1, s)$ such that $f^{s^{*}}(\widetilde{V})$ contains a critical point $c^{\prime} \in \operatorname{Crit}(f)$. It follows that $f^{s^{*}}(V) \subset f^{s^{*}}(\widetilde{V}) \subset \widetilde{B}\left(c^{\prime}, \tau\right)$. Let $\widetilde{V}^{*}, V^{*}$ be the connected components of $f^{-s^{*}}\left(\widetilde{B}\left(c^{\prime}, r \tau\right)\right.$ and $f^{-s^{*}}\left(\widetilde{B}\left(c^{\prime}, \tau\right)\right.$ containing $V$, respectively. If $f^{s^{*}}: \widetilde{V}^{*} \rightarrow$ $\widetilde{B}\left(c^{\prime}, r \tau\right)$ is diffeomorphic, the proof is completed with $s^{\prime}=s^{*}, c=c^{\prime}$ and $V^{\prime}=V^{*}$. Otherwise, repeating the argument above, we must stop within finitely many steps. 


\section{Proof of Theorem 1.4}

This section is devoted to the proof of Theorem 1.4. Throughout the rest of this section, let $\delta_{0}>0, \theta_{0} \in(0,1), \beta>0$ and let $\mathbf{r}:\left(0, \delta_{0}\right) \rightarrow(1,+\infty)$ be a function such that

$$
\sum_{n \gg 1} \mathbf{r}\left(\theta_{0}^{n}\right)^{-\beta}<+\infty
$$

and such that $f$ is backward contracting with function $\mathbf{r}$. Let $\Gamma$ and $\gamma$ be the functions defined in (2.1) and (2.2) respectively. In particular, $\gamma$ is well defined on $\left(0, \delta_{0}\right]$.

Observe that, for every $\delta \in\left(0, \delta_{0}\right]$, we have $\gamma(\delta) \geq \mathbf{r}(\delta)$. It follows that there is an integer $N \geq 1$ such that

$$
\sum_{n=N}^{+\infty} \gamma\left(\theta_{0}^{n}\right)^{-\beta}<+\infty
$$

By Lemma 2.7 we know that $f \in \mathrm{BC}(\infty)$. In view of Lemma 2.4, the rest of Theorem 1.4 is a direct consequence of the following proposition.

Proposition 3.1. Take $\alpha>0$ and $\vartheta \in(0,1)$. Assume that there exists an integer $N \geq 1$ such that $\sum_{n=N}^{+\infty} \gamma\left(\vartheta^{n}\right)^{-\alpha}<+\infty$. Then $\sum_{n=N}^{+\infty} \Gamma\left(\vartheta^{n}\right)^{-\alpha}<+\infty$.

The proof of this proposition, which is given at the end of this section, depends on several lemmas.

Lemma 3.2. For every $\delta \in\left(0, \delta_{0}\right]$, we have $\Gamma(\delta \gamma(\delta)) \geq \gamma(\delta)$.

Proof. Fix $\delta \in\left(0, \delta_{0}\right]$. If we let

$$
s(\delta):=\sup \{|V|: V \in \mathscr{P}(\delta \gamma(\delta))\},
$$

then by the definition of $\gamma(\delta)$ we have $s(\delta) \leq \delta$. It follows by the definition of $\Gamma(\delta)$ that

$$
\Gamma(\delta \gamma(\delta))=\inf \left\{\frac{\delta \gamma(\delta)}{|V|}: V \in \mathscr{P}(\delta \gamma(\delta))\right\}=\frac{\delta \gamma(\delta)}{s(\delta)} \geq \frac{\delta \gamma(\delta)}{\delta}=\gamma(\delta)
$$

This completes the proof.

Lemma 3.3. If $f \in \mathrm{BC}(\infty)$ then, for every $\theta \in(0,1)$, there exist an integer $N \geq 1$ and a constant $\varepsilon=\varepsilon(\theta)>0$ such that, for every $n \geq N$, we have $\gamma\left(\theta^{n+1}\right) \geq \varepsilon \gamma\left(\theta^{n}\right)$.

Proof. Put $\tau:=2^{1 / \ell_{\max }}-1$. Let $N \geq 1$ be an integer such that $\theta^{N} \leq \delta_{0}$, let $r_{1} \geq 4$ be sufficiently large so that

$$
\frac{1}{0.9}\left(\frac{1+\tau}{\tau}\right)^{2}\left(2 / r_{1}\right)^{1 / \ell_{\max }}<\theta
$$

and let $\tau_{0}$ be given by Lemma 2.8 with $r=r_{1}$. Moreover, let $\varepsilon>0$ be a sufficiently small constant so that $\varepsilon \gamma\left(\theta^{N}\right) \theta^{N}<\tau_{0}$ and $r_{1} \varepsilon \theta<2$. By Lemma 2.5, for every integer $n \geq N$, we have $\varepsilon \gamma\left(\theta^{n}\right) \theta^{n}<\tau_{0}$. To end the proof, by Lemma 2.8 it 
suffices to prove that, for every integer $n \geq N$, if $V$ is a connected component of $f^{-s}\left(\widetilde{B}\left(c, \varepsilon \gamma\left(\theta^{n}\right) \theta^{n+1}\right)\right)$ for some integer $s \geq 1$ and $c \in \operatorname{Crit}(f)$ containing a critical value and such that $\left.f^{s}: V \rightarrow \widetilde{B}\left(c, \varepsilon \gamma\left(\theta^{n}\right) \theta^{n+1}\right)\right)$ can be extended diffeomorphically onto $\left.\widetilde{B}\left(c, r_{1} \varepsilon \gamma\left(\theta^{n}\right) \theta^{n+1}\right)\right)$, then $|V|<\theta^{n+1}$. Fix such $V$. Let $V^{\prime}$ be the connected component of $f^{-s}\left(\widetilde{B}\left(c,\left(r_{1} \varepsilon \gamma\left(\theta^{n}\right) \theta^{n+1}\right) / 2\right)\right)$ containing $V$. Observe that both connected components of $\left.\widetilde{B}\left(c, r_{1} \varepsilon \gamma\left(\theta^{n}\right) \theta^{n+1}\right) \backslash \widetilde{B}\left(c,\left(r_{1} \varepsilon \gamma\left(\theta^{n}\right) \theta^{n+1}\right) / 2\right)\right)$ have length at least $\tau\left|\widetilde{B}\left(c,\left(r_{1} \varepsilon \gamma\left(\theta^{n}\right) \theta^{n+1}\right) / 2\right)\right|$. Therefore, by Lemma 2.1,

$$
\frac{\left|\widetilde{B}\left(c,\left(r_{1} \varepsilon \gamma\left(\theta^{n}\right) \theta^{n+1}\right) / 2\right)\right|}{\left|V^{\prime}\right|} \leq \frac{1}{0.9}\left(\frac{1+\tau}{\tau}\right)^{2} \frac{\left|\widetilde{B}\left(c, \varepsilon \gamma\left(\theta^{n}\right) \theta^{n+1}\right)\right|}{|V|} .
$$

Noticing that $\left|V^{\prime}\right| \leq \theta^{n}$ by the backward contracting property and our choice of $\varepsilon$,

$$
|V| \leq \frac{1}{0.9}\left(\frac{1+\tau}{\tau}\right)^{2}\left(\frac{2}{r_{1}}\right)^{1 / \ell_{c}} \theta^{n}<\theta^{n+1} .
$$

The proof of the lemma is thus completed.

Proof of Proposition 3.1. For each integer $n \geq 1$, let $l(n)$ be the positive integer such that $\vartheta^{l(n)} \leq \vartheta^{n} \gamma\left(\vartheta^{n}\right)<\vartheta^{l(n)-1}$. By the assumption,

$$
\sum_{n=N}^{+\infty} \vartheta^{\alpha(n-l(n))}<\sum_{n=N}^{+\infty}\left(\vartheta \gamma\left(\vartheta^{n}\right)\right)^{-\alpha}<+\infty
$$

By Lemma 2.5, we have $\vartheta^{n} \gamma\left(\vartheta^{n}\right) \geq \vartheta^{n+1} \gamma\left(\vartheta^{n+1}\right)$. Together with the definition of $l(n)$, we have $\vartheta^{l(n+1)}<\vartheta^{l(n)-1}$. This gives us $l(n) \leq l(n+1)$. It follows that $l(n)$ is an increasing function of $n$. Since by the assumption $\omega(\operatorname{Crit}(f)) \cap \operatorname{Crit}(f) \neq \emptyset$, we have $l(n) \rightarrow+\infty$ as $n \rightarrow+\infty$.

Claim. There exists a constant $N_{0} \geq 1$ such that, for every $n \geq N$, we have $l(n+1) \leq$ $l(n)+N_{0}$.

In fact, by Lemma 2.7, we have $f \in \mathrm{BC}(\infty)$. Therefore, by Lemma 3.3 there exists $K$ such that, for every integer $n \geq N$, we have $\gamma\left(\vartheta^{n+1}\right) \geq \vartheta^{K} \gamma\left(\vartheta^{n}\right)$. It follows by the definition of $l(n)$ that $l(n+1) \leq l(n)+K+2$. The claim is proved with $N_{0}=K+2$.

On the other hand, by Lemmas 2.2 and 3.2 and the definition of $l(n)$,

$$
\vartheta^{l(n)} \Gamma\left(\vartheta^{l(n)}\right)^{-1} \leq \vartheta^{n} \gamma\left(\vartheta^{n}\right) \Gamma\left(\vartheta^{n} \gamma\left(\vartheta^{n}\right)\right)^{-1} \leq \vartheta^{n} \gamma\left(\vartheta^{n}\right) \gamma\left(\vartheta^{n}\right)^{-1}=\vartheta^{n}
$$

It follows that $\Gamma\left(\vartheta^{l(n)}\right)^{-1} \leq \vartheta^{n-l(n)}$. Therefore, together with (3.1),

$$
\sum_{n=N}^{+\infty} \Gamma\left(\vartheta^{l(n)}\right)^{-\alpha} \leq \sum_{n=N}^{+\infty} \vartheta^{\alpha(n-l(n))}<+\infty
$$

By Lemma 2.2 again, for every $i \geq 1$,

$$
\vartheta^{l(n)+i} \Gamma\left(\vartheta^{l(n)+i}\right)^{-1} \leq \vartheta^{l(n)} \Gamma\left(\vartheta^{l(n)}\right)^{-1} .
$$


Hence, for every $i \geq 1$,

$$
\sum_{n=N}^{+\infty} \Gamma\left(\vartheta^{l(n)+i}\right)^{-\alpha} \leq \sum_{n=N}^{+\infty} \vartheta^{-i \alpha} \Gamma\left(\vartheta^{l(n)}\right)^{-\alpha}<+\infty .
$$

Together with (3.2) and the claim above, this proves that $\sum_{n=N}^{+\infty} \Gamma\left(\vartheta^{n}\right)^{-\alpha}<+\infty$.

\section{Acknowledgements}

We would like to thank Weixiao Shen for his helpful comments and suggestions. We would also like to thank the anonymous referee for valuable comments and suggestions.

\section{References}

[1] H. Bruin, J. Rivera-Letelier, W. Shen and S. van Strien, 'Large derivatives, backward contraction and invariant densities for interval maps', Invent. Math. 172 (2008), 509-533.

[2] P. Collet and J. Eckmann, 'Positive Liapunov exponents and absolute continuity for maps of the interval', Ergod. Th. E Dynam. Sys. 3(1) (1983), 13-46.

[3] H. Li and W. Shen, 'Dimensions of rational maps satisfying the backward contraction property', Fund. Math. 198(2) (2008), 165-176.

[4] H. Li and W. Shen, 'Topological invariance of a strong summability condition in one-dimensional dynamics', Int. Math. Res. Not. 8 (2013), 1783-1799.

[5] T. Nowicki and S. van Strien, 'Invariant measures exist under a summability condition for unimodal maps', Invent. Math. 105(1) (1991), 123-136.

[6] J. Rivera-Letelier, 'A connecting lemma for rational maps satisfying a no growth condition', Ergod. Th. E Dynam. Sys. 27 (2007), 595-636.

HUAIBIN LI, School of Mathematics and Information Science, Henan University, Kaifeng 475004, PR China

e-mail: lihbmath@henu.edu.cn 